\title{
A note on inequalities and critical values of fuzzy rough variables
}

\author{
Mingxuan Zhao, Jing Liu and Ke Wang*
}

"Correspondence: ke@shu.edu.cn School of Management, Shanghai University, Shanghai, 200444, China

\begin{abstract}
A fuzzy rough variable is defined as a rough variable on the universal set of fuzzy variables, or a rough variable taking 'fuzzy variable' values. In order to further discuss the mathematical properties of fuzzy rough variables, this paper extends some inequalities to the context of fuzzy rough theory based on the chance measure and the expected value operator, involving the Markov inequality, the Chebyshev inequality, the Hölder inequality, the Minkowski inequality, and the Jensen inequality. After that, linearity, monotonicity, and continuity of critical values of fuzzy rough variables are also investigated.
\end{abstract}

Keywords: fuzzy rough variable; expected value operator; chance measure; critical value

\section{Introduction}

Fuzzy set theory has been well developed and applied in a wide variety of real problems since it was proposed in 1965 by Zadeh [1]. Kaufmann [2] first introduced fuzzy variable as a fuzzy set of real numbers to describe fuzzy phenomena. By means of a mathematical way, a fuzzy variable was defined as a function from a possibility space to the set of real numbers by Liu [3]. Now, fuzzy set theory has been proved to be an excellent tool and one of the most successful approaches to the issue of how to understand and manipulate imperfect knowledge.

On the other hand, in order to deal with vague description of objects, rough set theory was initialized by Pawlak [4] in 1982, which provides a new powerful mathematical approach to handling imperfect knowledge in the real world. A fundamental assumption in this theory is that objects are perceived through, and thus can be represented by, available information on their attributes, but such information may not be sufficient to characterize these objects exactly. One way is approximating a set by other sets. Thus a rough set may be defined by a pair of crisp sets which give the lower and upper approximations of the original set. Liu [5] defined a rough variable to be a measurable function from a rough space to the set of real numbers and gave the definition of the lower and upper approximations of the rough variable.

With the development of fuzzy set theory and rough set theory, it is generally accepted that these two theories are related but distinct and complementary with each other. Thus many researchers began to consider the combination of the two theories. For example, in the real world, sometimes it is not easy to describe a fuzzy event by a precise fuzzy set, but

(c) 2015 Zhao et al. This article is distributed under the terms of the Creative Commons Attribution 4.0 International License (http://creativecommons.org/licenses/by/4.0/), which permits unrestricted use, distribution, and reproduction in any medium, provided you give appropriate credit to the original author(s) and the source, provide a link to the Creative Commons license, and indicate if changes were made. 
the lower and upper approximations of the fuzzy set of a fuzzy event can be given, which can be seen as a fuzzy rough variable. In 1990, Dubois and Prade [6] initially proposed the concept of fuzzy rough sets by constructing a pair of upper and lower approximation operators of fuzzy sets with respect to a fuzzy similarity relation by means of the $t$-norm Min and its dual conorm Max. A fuzzy rough variable, very different from the fuzzy rough set introduced by Dubois and Prade [6], was defined by Liu [5] as a measurable function from a rough space to the set of fuzzy variables. In other words, a fuzzy rough variable is a rough variable defined on the universal set of fuzzy variables, or a rough variable taking 'fuzzy variable' values. By now, the fuzzy rough theory has been studied in both theoretical and practical perspectives, for instance, the generalized definition of a fuzzy rough set [7, $8]$, a new definition for the lower and upper approximations $[9,10]$, fuzzy rough attribute (or feature) selection $[11,12]$, as well as its applications in data reduction and classification [13-15], complex systems monitoring [16], neural networks [17], and so on.

It is well known that there are some inequalities in probability theory such as the Markov inequality, the Chebyshev inequality, the Jensen inequality, the Hölder inequality, and the Minkowski inequality, which make an important contribution to the development of probability theory in both theories and applications. On the basis of these inequalities, in possibility and rough theory, Liu [18] proved that these analogous inequalities hold both for fuzzy variables and rough variables. Moreover, Yang and Liu [19] also proved these inequalities for fuzzy random variables. As an extension of these researches, it is necessary to study these inequalities in the context of fuzzy rough theory. Therefore, in the present paper, some inequalities are presented for fuzzy rough variables, and some properties of critical values of fuzzy rough variables are also proved.

The rest of this paper is organized as follows. In Section 2, we first review some basic knowledge of fuzzy variables, rough variables, and fuzzy rough variables involving the chance measure and the expected value operator. Some inequalities for fuzzy rough variables are presented in Section 3. Section 4 introduces definitions of the $(\gamma, \delta)$-optimistic value and the $(\gamma, \delta)$-pessimistic value, and it discusses the linearity, monotonicity and continuity of the critical values to explore the mathematical properties of fuzzy rough variables.

\section{Preliminaries}

In this section, we recall some concepts and properties of fuzzy variables, rough variables, and fuzzy rough variables, which will be applied in the following sections.

\subsection{Fuzzy variable}

In order to measure a fuzzy event, Zadeh $[20,21]$ proposed the concepts of possibility measure and necessity measure in 1978 and 1979, respectively. Subsequently, possibility theory was developed by many researchers such as Dubois and Prade [22, 23]. Liu and Liu [24] presented the concept of credibility measure in 2002 on the basis of possibility measure and necessity measure, and then a complete axiomatic foundation of credibility theory was developed by Liu [3].

Definition 1 ( $\mathrm{Liu}$ [5]) Let $\Theta$ be a nonempty set, $\mathcal{P}(\Theta)$ the power set of $\Theta$, and Pos a possibility measure. The triplet $(\Theta, \mathcal{P}(\Theta)$, Pos) is called a possibility space. A fuzzy variable is defined as a function from a possibility space $(\Theta, \mathcal{P}(\Theta)$, Pos) to the set of real numbers. 
Definition 2 (Liu and Liu [24]) Let $(\Theta, \mathcal{P}(\Theta)$, Pos) be a possibility space, and $A$ a set in $\mathcal{P}(\Theta)$. Then the credibility measure of $A$ is defined by

$$
\operatorname{Cr}\{A\}=\frac{1}{2}(\operatorname{Pos}\{A\}+\operatorname{Nec}\{A\})
$$

where Pos and Nec represent the possibility measure [20] and the necessity measure [21], respectively.

Example 1 Let us consider a trapezoidal fuzzy variable $\xi=\left(r_{1}, r_{2}, r_{3}, r_{4}\right)$. Then we have

$$
\begin{aligned}
& \operatorname{Pos}\{\xi<0\}= \begin{cases}1, & \text { if } r_{2} \leq 0, \\
\frac{r_{1}}{r_{1}-r_{2}}, & \text { if } r_{1} \leq 0 \leq r_{2}, \\
0, & \text { otherwise, }\end{cases} \\
& \operatorname{Nec}\{\xi<0\}= \begin{cases}1, & \text { if } r_{4} \leq 0, \\
\frac{r_{3}}{r_{3}-r_{4}}, & \text { if } r_{3} \leq 0 \leq r_{4}, \\
0, & \text { otherwise, }\end{cases} \\
& \operatorname{Cr}\{\xi<0\}= \begin{cases}1, & \text { if } r_{4} \leq 0, \\
\frac{2 r_{3}-r_{4}}{2\left(r_{3}-r_{4}\right)}, & \text { if } r_{3} \leq 0 \leq r_{4}, \\
\frac{1}{2}, & \text { if } r_{2} \leq 0 \leq r_{3}, \\
\frac{r_{1}}{2\left(r_{1}-r_{2}\right)}, & \text { if } r_{1} \leq 0 \leq r_{2}, \\
0, & \text { otherwise. }\end{cases}
\end{aligned}
$$

Definition 3 (Liu and Gao [25]) The fuzzy variables $\xi_{1}, \xi_{2}, \ldots, \xi_{n}$ are said to be independent if

$$
\operatorname{Cr}\left\{\xi_{i} \in B_{i}, i=1,2, \ldots, n\right\}=\min _{1 \leq i \leq n} \operatorname{Cr}\left\{\xi_{i} \in B_{i}\right\}
$$

for any Borel sets $B_{1}, B_{2}, \ldots, B_{n}$ of real numbers.

Theorem 1 (Liu and Gao [25]) The fuzzy variables $\xi_{1}, \xi_{2}, \ldots, \xi_{n}$ are independent if and only if

$$
\operatorname{Cr}\left\{\bigcup_{i=1}^{n}\left\{\xi_{i} \in B_{i}\right\}\right\}=\max _{1 \leq i \leq n} \operatorname{Cr}\left\{\xi_{i} \in B_{i}\right\}
$$

for any Borel sets $B_{1}, B_{2}, \ldots, B_{n}$ of real numbers.

Definition 4 (Liu and Liu [24]) Let $\xi$ be a fuzzy variable. Then the expected value of $\xi$ is defined by

$$
E^{\mathrm{f}}[\xi]=\int_{0}^{+\infty} \operatorname{Cr}\{\xi \geq r\} \mathrm{d} r-\int_{-\infty}^{0} \operatorname{Cr}\{\xi \leq r\} \mathrm{d} r
$$

provided that at least one of the two integrals is finite, where $E^{\mathrm{f}}$ denotes the expected value operator of fuzzy variables. 
Example 2 Let $\xi$ be a fuzzy variable with a membership function

$$
\mu(x)= \begin{cases}1, & \text { if } x \in[a, b], \\ 0, & \text { otherwise }\end{cases}
$$

Then the expected value of $\xi$ is $E^{\mathrm{f}}[\xi]=\frac{1}{2}(a+b)$.

Example 3 The expected value of a trapezoidal fuzzy variable $\xi=\left(r_{1}, r_{2}, r_{3}, r_{4}\right)$ is

$$
E^{\mathrm{f}}[\xi]=\frac{1}{4}\left(r_{1}+r_{2}+r_{3}+r_{4}\right)
$$

Theorem 2 (Liu [24]) Let $\xi$ and $\eta$ be fuzzy variables with finite expected values. Then, for any real numbers $a$ and $b$, we have

$$
E^{\mathrm{f}}[a \xi+b \eta]=a E^{\mathrm{f}}[\xi]+b E^{\mathrm{f}}[\eta]
$$

\subsection{Rough variable}

Rough set theory, initialized by Pawlak [4], has been proved to be an excellent mathematical tool to deal with vague description of objects. In order to provide an axiomatic theory to describe rough variables, Liu [5] gave some definitions about rough set theory as follows.

Definition 5 (Liu [5]) Let $\Lambda$ be a nonempty set, $\mathcal{A}$ a $\sigma$-algebra of subset of $\Lambda, \Delta$ an element in $\mathcal{A}$, and $\pi$ a set function satisfying the following four axioms,

Axiom 1. $\pi\{\Lambda\}<+\infty$;

Axiom 2. $\pi\{\Delta\}>0$;

Axiom 3. $\pi\{A\} \geq 0$ for any $A \in \mathcal{A}$;

Axiom 4 . for every countable sequence of mutually disjoint events $\left\{A_{i}\right\}_{i=1}^{\infty}$, we have

$$
\pi\left\{\bigcup_{i=1}^{\infty} A_{i}\right\}=\sum_{i=1}^{\infty} \pi\left\{A_{i}\right\} .
$$

Then $(\Lambda, \triangle, \mathcal{A}, \pi)$ is called a rough space.

Definition 6 (Liu [5]) A rough variable is a measurable function $\xi$ from the rough space $(\Lambda, \Delta, \mathcal{A}, \pi)$ to the set of real numbers. That is, for every Borel set $B$ of $\Re$, we have

$$
\{\lambda \in \Lambda \mid \xi(\lambda) \in B\} \in \mathcal{A} .
$$

The lower and upper approximations of the rough variable $\xi$ are then defined as follows:

$$
\underline{\xi}=\{\xi(\lambda) \mid \lambda \in \Delta\}, \quad \bar{\xi}=\{\xi(\lambda) \mid \lambda \in \Lambda\} .
$$

Definition 7 (Liu [5]) Let $(\Lambda, \Delta, \mathcal{A}, \pi)$ be a rough space. Then the upper trust of an event $A$ is defined by

$$
\overline{\operatorname{Tr}}\{A\}=\frac{\pi\{A\}}{\pi\{\Lambda\}}
$$


the lower trust of the event $A$ is defined by

$$
\underline{\operatorname{Tr}}\{A\}=\frac{\pi\{A \cap \Delta\}}{\pi\{\Delta\}}
$$

and the trust of the event $A$ is defined by

$$
\operatorname{Tr}\{A\}=\frac{1}{2}(\overline{\operatorname{Tr}}\{A\}+\underline{\operatorname{Tr}}\{A\}) .
$$

Example 4 Let $\xi=([a, b],[c, d])$ be a rough variable with $c \leq a<b \leq d$ representing the identity function $\xi(\lambda)=\lambda$ from the rough space $(\Lambda, \Delta, \mathcal{A}, \pi)$ to the set of real numbers, where $\Lambda=\{c \leq \lambda \leq d\}, \Delta=\{a \leq \lambda \leq b\}$, and $\pi$ is the Lebesgue measure, which is a standard method that gives the subsets of a Euclidean space a length, area, or volume. In this case, $\pi$ measures the length of a set. For example, if a set $A=[a, b]$, then $\pi\{A\}=b-a$. According to Definitions 6 and 7, we can obtain the upper trust, the lower trust, and trust of the event $\{\xi \leq 0\}$ as follows:

$$
\begin{aligned}
& \overline{\operatorname{Tr}}\{\xi \leq 0\}= \begin{cases}0, & \text { if } c \geq 0, \\
\frac{c}{c-d}, & \text { if } c \leq 0 \leq d, \\
1, & \text { if } d \leq 0,\end{cases} \\
& \underline{\operatorname{Tr}}\{\xi \leq 0\}= \begin{cases}0, & \text { if } a \geq 0, \\
\frac{a}{a-b}, & \text { if } a \leq 0 \leq b, \\
1, & \text { if } b \leq 0,\end{cases} \\
& \operatorname{Tr}\{\xi \leq 0\}= \begin{cases}0, & \text { if } c \geq 0, \\
\frac{c}{2(c-d)}, & \text { if } c \leq 0 \leq a, \\
\frac{2 a c-a d-b c}{2(b-a)(d-c)}, & \text { if } a \leq 0 \leq b, \\
\frac{d-2 c}{2(d-c)}, & \text { if } b \leq 0 \leq d, \\
1, & \text { if } d \leq 0 .\end{cases}
\end{aligned}
$$

When $[a, b]=[c, d]$, we have $\Lambda=\Delta$, and $\overline{\operatorname{Tr}}\{\xi \leq 0\}=\underline{\operatorname{Tr}}\{\xi \leq 0\}$. It follows that

$$
\operatorname{Tr}\{\xi \leq 0\}= \begin{cases}0, & \text { if } a \geq 0, \\ \frac{a}{a-b}, & \text { if } a \leq 0 \leq b, \\ 1, & \text { if } b \leq 0 .\end{cases}
$$

Definition 8 (Liu [5]) Let $\xi$ be a rough variable. Then the expected value of $\xi$ is defined by

$$
E^{\mathrm{r}}[\xi]=\int_{0}^{+\infty} \operatorname{Tr}\{\xi \geq r\} \mathrm{d} r-\int_{-\infty}^{0} \operatorname{Tr}\{\xi \leq r\} \mathrm{d} r
$$

provided that at least one of the two integrals is finite, where $E^{\mathrm{r}}$ denotes the expected value operator of rough variables.

Example 5 Let $\xi=([a, b],[c, d])$ be a rough variable with $c \leq a<b \leq d$. We then have

$$
E^{\mathrm{r}}[\xi]=\frac{1}{4}(a+b+c+d)
$$


Especially, when $[a, b]=[c, d]$, the rough variable $\xi$ degenerates to an interval number $[a, b]$. Then we have

$$
E^{\mathrm{r}}[\xi]=\frac{1}{2}(a+b)
$$

Theorem 3 (Liu [3]) Let $\xi$ and $\eta$ be rough variables with finite expected values. Then, for any real numbers $a$ and $b$, we have

$$
E^{\mathrm{r}}[a \xi+b \eta]=a E^{\mathrm{r}}[\xi]+b E^{\mathrm{r}}[\eta]
$$

\subsection{Fuzzy rough variable}

Fuzzy rough variables have been defined in several ways. In this paper, we adopt the definition introduced by Liu [5] as follows.

Definition 9 (Liu [5]) A fuzzy rough variable is a function $\xi$ from a rough space $(\Lambda, \Delta, \mathcal{A}, \pi)$ to the set of fuzzy variables such that $\operatorname{Pos}\{\xi(\lambda) \in B\}$ is a measurable function of $\lambda$ for any Borel set $B$ of $\Re$.

Example 6 Let $\xi=(\rho, \rho+1, \rho+2)$ with $\rho=([2,4],[0,6])$, where the triple $(a, b, c)$ with real numbers $a \leq b \leq c$ denotes a triangular fuzzy variable and $\rho$ is a rough variable, then $\xi$ is a fuzzy rough variable.

Theorem 4 (Liu [3]) Assume that $\xi$ is a fuzzy rough variable. Then, for any Borel set B of $\Re$, we have

(a) the possibility $\operatorname{Pos}\{\xi(\lambda) \in B\}$ is a rough variable;

(b) the necessity $\operatorname{Nec}\{\xi(\lambda) \in B\}$ is a rough variable;

(c) the credibility $\operatorname{Cr}\{\xi(\lambda) \in B\}$ is a rough variable.

Definition 10 (Liu [5]) Let $\xi$ be a fuzzy rough variable. Then its expected value is defined by

$$
E[\xi]=\int_{0}^{\infty} \operatorname{Tr}\left\{\lambda \in \Lambda \mid E^{\mathrm{f}}[\xi(\lambda)] \geq r\right\} \mathrm{d} r-\int_{-\infty}^{0} \operatorname{Tr}\left\{\lambda \in \Lambda \mid E^{\mathrm{f}}[\xi(\lambda)] \leq r\right\} \mathrm{d} r
$$

provided that at least one of the two integrals is finite, where $E^{\mathrm{f}}$ is the expected value operator of fuzzy variables.

Theorem 5 (Liu [5]) Assume that $\xi$ and $\eta$ are fuzzy rough variables with finite expected values. If $\xi(\lambda)$ and $\eta(\lambda)$ are independent fuzzy variables for each $\lambda$, then, for any real numbers $a$ and $b$, we have

$$
E[a \xi+b \eta]=a E[\xi]+b E[\eta]
$$

Definition 11 (Liu [5]) Let $\xi$ be a fuzzy rough variable with finite expected value $E[\xi]$. The variance of $\xi$ is defined as $V[\xi]=E\left[(\xi-E[\xi])^{2}\right]$. Then the square root of $V[\xi]$ is called the standard deviation of $\xi$. 
Definition 12 (Liu [5]) Let $\xi$ be a fuzzy rough variable, and $B$ a Borel set of $\Re$. Then the chance of a fuzzy rough event $\xi \in B$ is a function from $(0,1]$ to $[0,1]$, defined as

$$
\operatorname{Ch}\{\xi \in B\}(\alpha)=\sup \{\beta \mid \operatorname{Tr}\{\lambda \in \Lambda \mid \operatorname{Cr}\{\xi(\lambda) \in B\} \geq \beta\} \geq \alpha\}
$$

which can also be written as follows:

$$
\operatorname{Ch}\{\xi \in B\}(\alpha)=\sup _{\operatorname{Tr}\{A\} \geq \alpha} \inf _{\lambda \in A} \operatorname{Cr}\{\xi(\lambda) \in B\}
$$

Definition 13 (Liu [3]) Let $\xi$ be a fuzzy rough variable, and $B$ a Borel set of $\Re$. For any real number $\alpha \in(0,1]$, the $\alpha$-chance of a fuzzy rough event $\xi \in B$ is defined as the value of chance at $\alpha$, i.e., $\mathrm{Ch}\{\xi \in B\}(\alpha)$, where $\mathrm{Ch}$ denotes the chance measure.

\section{Inequalities of fuzzy rough variables}

Some inequalities, including the Markov inequality, the Chebyshev inequality, the Hölder inequality, the Minkowski inequality, and the Jensen inequality, analogous to those in probability theory, have been proved to hold both for fuzzy variables and rough variables by Liu [18]. In this section, these inequalities are proved for fuzzy rough variables.

Theorem 6 Let $\xi$ be a fuzzy rough variable, and $f$ a nonnegative measurable function. If $f$ is even and increasing on $[0, \infty)$, then, for any given number $t>0$ and $\alpha \in(0,1]$, we have

$$
\operatorname{Ch}\{|\xi| \geq t\}(\alpha) \leq \frac{E[f(\xi)]}{\alpha f(t)}
$$

Proof For a fuzzy variable $\tau$, the following inequality has been proved by Liu [18].

$$
\operatorname{Cr}\{|\tau| \geq t\} \leq \frac{E^{\mathrm{f}}[f(\tau)]}{f(t)}, \quad \forall t>0,
$$

on condition that $f$ is a nonnegative function and it is even and increasing on $[0, \infty)$. Since $\xi(\lambda)$ is a fuzzy variable, for any $\lambda \in \Lambda$, it follows that

$$
\operatorname{Cr}\{|\xi(\lambda)| \geq t\} \leq \frac{E^{\mathrm{f}}[f(\xi(\lambda))]}{f(t)}
$$

for each $\lambda$. Especially, for any set $A$ with $\operatorname{Tr}\{A\} \geq \alpha$, we have

$$
\inf _{\lambda^{*} \in A} \operatorname{Cr}\left\{\left|\xi\left(\lambda^{*}\right)\right| \geq t\right\} \leq \frac{E^{\mathrm{f}}[f(\xi(\lambda))]}{f(t)}, \quad \forall \lambda \in A .
$$

It follows from the nonnegativity of $f$ that

$$
\begin{aligned}
\frac{E[f(\xi)]}{f(t)} & =\frac{1}{f(t)} \int_{0}^{+\infty} \operatorname{Tr}\left\{\lambda \in \Lambda \mid E^{\mathrm{f}}[f(\xi(\lambda))] \geq r\right\} \mathrm{d} r \\
& \geq \frac{1}{f(t)} \int_{0}^{+\infty} \operatorname{Tr}\left\{\lambda \in A \mid E^{\mathrm{f}}[f(\xi(\lambda))] \geq r\right\} \mathrm{d} r \\
& \geq \int_{0}^{+\infty} \operatorname{Tr}\left\{\lambda \in A \mid \inf _{\lambda^{*} \in A} \operatorname{Cr}\left\{\left|\xi\left(\lambda^{*}\right)\right| \geq t\right\} \geq r\right\} \mathrm{d} r
\end{aligned}
$$




$$
\begin{aligned}
& =\inf _{\lambda^{*} \in A} \operatorname{Cr}\left\{\left|\xi\left(\lambda^{*}\right)\right| \geq t\right\} \cdot \operatorname{Tr}\{A\} \\
& \geq \inf _{\lambda^{*} \in A} \operatorname{Cr}\left\{\left|\xi\left(\lambda^{*}\right)\right| \geq t\right\} \cdot \alpha
\end{aligned}
$$

Therefore, we have

$$
\inf _{\lambda^{*} \in A} \operatorname{Cr}\left\{\left|\xi\left(\lambda^{*}\right)\right| \geq t\right\} \leq \frac{E[f(\xi)]}{\alpha f(t)}
$$

It follows from the arbitrariness of $A$ that

$$
\sup _{\operatorname{Tr}\{A\} \geq \alpha} \inf _{\lambda^{*} \in A} \operatorname{Cr}\left\{\left|\xi\left(\lambda^{*}\right)\right| \geq t\right\} \leq \frac{E[f(\xi)]}{\alpha f(t)} .
$$

That is,

$$
\operatorname{Ch}\{|\xi| \geq t\}(\alpha) \leq \frac{E[f(\xi)]}{\alpha f(t)}
$$

The proof is completed.

On the basis of the inequality presented in Theorem 6, the well-known Markov inequality and the Chebyshev inequality in probability theory are proved in the context of fuzzy rough theory as follows, which can be seen as special cases of Theorem 6 .

Theorem 7 (Markov inequality) Let $\xi$ be a fuzzy rough variable. Then, for any given numbers $t>0, p>0$, and $\alpha \in(0,1]$, we have

$$
\operatorname{Ch}\{|\xi| \geq t\}(\alpha) \leq \frac{E\left[|\xi|^{p}\right]}{\alpha t^{p}} .
$$

Proof It is a special case of Theorem 6 when $f(x)=|x|^{p}$.

Theorem 8 (Chebyshev inequality) Let $\xi$ be a fuzzy rough variable whose variance $V[\xi]$ exists. Then, for any given numbers $t>0$ and $\alpha \in(0,1]$, we have

$$
\operatorname{Ch}\{|\xi-E[\xi]| \geq t\}(\alpha) \leq \frac{V[\xi]}{\alpha t^{2}}
$$

Proof It is a special case of Theorem 6 when the fuzzy rough variable $\xi$ is replaced with $\xi-E[\xi]$ and $f(x)=x^{2}$.

The Markov inequality gives an upper bound for the $\alpha$-chance that the absolute value of a fuzzy rough variable is greater than or equal to some positive constant, whereas the Chebyshev inequality describes to what extent the values taken by a fuzzy rough variable deviate from its expected value. Both of them state important properties of a fuzzy rough variable.

For instance, the following conclusion can be deduced from the Chebyshev inequality immediately, which implies that the $\alpha$-chance that the values taken by an arbitrary fuzzy rough variable with finite expected value exceed $k(k>0)$ standard deviations away from its mean (i.e., expected value) is no more than $\frac{1}{\alpha k^{2}}$. 
Example 7 Let $\xi$ be a fuzzy rough variable with finite expected value $e$ and standard deviation $\sigma$. It follows from Theorem 8 that

$$
\operatorname{Ch}\{|\xi-e| \geq k \sigma\}(\alpha) \leq \frac{V[\xi]}{\alpha(k \sigma)^{2}}=\frac{1}{\alpha k^{2}}, \quad \forall \alpha \in(0,1], k>0 .
$$

Theorem 9 (Hölder inequality) Let $p$ and $q$ be two positive real numbers with $1 / p+1 / q=1$, $\xi$ and $\eta$ two fuzzy rough variables with $E\left[|\xi|^{p}\right]<\infty$ and $E\left[|\eta|^{q}\right]<\infty$. If the fuzzy variables $\xi(\lambda)$ and $\eta(\lambda)$ are independent for each $\lambda \in \Lambda$, then we have

$$
E[|\xi \eta|] \leq \sqrt[p]{E\left[|\xi|^{p}\right]} \sqrt[q]{E\left[|\eta|^{q}\right]}
$$

Proof The inequality holds trivially if at least one of $\xi$ and $\eta$ is zero a.s. Now we assume $E\left[|\xi|^{p}\right]>0$ and $E\left[|\eta|^{q}\right]>0$. It is easy to prove that the function $f(x, y)=\sqrt[p]{x} \sqrt[q]{y}$ is a concave function on $D=\{(x, y) \mid x \geq 0, y \geq 0\}$. Thus, for any point $\left(x_{0}, y_{0}\right)$ with $x_{0}>0$ and $y_{0}>0$, there exist two real numbers $a$ and $b$ such that

$$
f(x, y)-f\left(x_{0}, y_{0}\right) \leq a\left(x-x_{0}\right)+b\left(y-y_{0}\right), \quad \forall(x, y) \in D .
$$

Letting $x=|\xi|^{p}, y=|\eta|^{q}, x_{0}=E\left[|\xi|^{p}\right]$, and $y_{0}=E\left[|\eta|^{q}\right]$, we have

$$
f\left(|\xi|^{p},|\eta|^{q}\right)-f\left(E\left[|\xi|^{p}\right], E\left[|\eta|^{q}\right]\right) \leq a\left(|\xi|^{p}-E\left[|\xi|^{p}\right]\right)+b\left(|\eta|^{q}-E\left[|\eta|^{q}\right]\right) .
$$

Taking the expected values on both sides, we obtain

$$
E\left[f\left(|\xi|^{p},|\eta|^{q}\right)\right] \leq f\left(E\left[|\xi|^{p}\right], E\left[|\eta|^{q}\right]\right)
$$

Hence the inequality (31) holds.

As a special case of the Hölder inequality with $p=q=2$, the Cauchy inequality can be obtained as follows, which is widely used for dealing with some mathematical problems.

Example 8 (Cauchy inequality) Let $\xi$ and $\eta$ be two fuzzy rough variables with $E\left[\xi^{2}\right]<\infty$ and $E\left[\eta^{2}\right]<\infty$. If the fuzzy variables $\xi(\lambda)$ and $\eta(\lambda)$ are independent for each $\lambda \in \Lambda$, then it follows from Theorem 9 that

$$
E[|\xi \eta|] \leq \sqrt{E\left[\xi^{2}\right] \cdot E\left[\eta^{2}\right]} .
$$

Theorem 10 (Minkowski inequality) Let $p$ be a real number with $1 \leq p<\infty$, $\xi$, and $\eta$ two fuzzy rough variables with $E\left[|\xi|^{p}\right]<\infty$ and $E\left[|\eta|^{p}\right]<\infty$. If the fuzzy variables $\xi(\lambda)$ and $\eta(\lambda)$ are independent for each $\lambda \in \Lambda$, then we have

$$
\sqrt[p]{E\left[|\xi+\eta|^{p}\right]} \leq \sqrt[p]{E\left[|\xi|^{p}\right]}+\sqrt[p]{E\left[|\eta|^{p}\right]}
$$

Proof The inequality holds trivially if at least one of $\xi$ and $\eta$ is zero a.s. Now we assume $E\left[|\xi|^{p}\right]>0$ and $E\left[|\eta|^{p}\right]>0$. It is easy to prove that the function $f(x, y)=(\sqrt[p]{x}+\sqrt[p]{y})^{p}$ is a 
concave function on $D=\{(x, y) \mid x \geq 0, y \geq 0\}$. Thus, for any point $\left(x_{0}, y_{0}\right)$ with $x_{0}>0$ and $y_{0}>0$, there exist two real numbers $a$ and $b$ such that

$$
f(x, y)-f\left(x_{0}, y_{0}\right) \leq a\left(x-x_{0}\right)+b\left(y-y_{0}\right), \quad \forall(x, y) \in D
$$

Letting $x=|\xi|^{p}, y=|\eta|^{p}, x_{0}=E\left[|\xi|^{p}\right]$, and $y_{0}=E\left[|\eta|^{p}\right]$, we have

$$
f\left(|\xi|^{p},|\eta|^{p}\right)-f\left(E\left[|\xi|^{p}\right], E\left[|\eta|^{p}\right]\right) \leq a\left(|\xi|^{p}-E\left[|\xi|^{p}\right]\right)+b\left(|\eta|^{p}-E\left[|\eta|^{p}\right]\right) .
$$

Taking the expected values on both sides, we obtain

$$
E\left[f\left(|\xi|^{p},|\eta|^{p}\right)\right] \leq f\left(E\left[|\xi|^{p}\right], E\left[|\eta|^{p}\right]\right) .
$$

Hence the inequality (32) holds.

Theorem 11 (Jensen inequality) Let $\xi$ be a fuzzy rough variable, and $f$ a convex function. If $E[\xi]$ and $E[f(\xi)]$ are finite, then

$$
f(E[\xi]) \leq E[f(\xi)]
$$

Especially, when $f(x)=|x|^{p}$ and $p \geq 1$, we have $|E[\xi]|^{p} \leq E\left[|\xi|^{p}\right]$.

Proof Since $f$ is a convex function, for each $y$, there exists a number $k$ such that $f(x)-f(y) \geq$ $k \cdot(x-y)$. Replacing $x$ with $\xi$ and $y$ with $E[\xi]$, we obtain

$$
f(\xi)-f(E[\xi]) \geq k \cdot(\xi-E[\xi])
$$

Taking the expected values on both sides, we have

$$
E[f(\xi)]-f(E[\xi]) \geq k \cdot(E[\xi]-E[\xi])=0 .
$$

Hence the inequality (33) holds.

The Jensen inequality gives a lower bound for the expected value of a convex function of a fuzzy rough variable. On the basis of the Jensen inequality, some other inequalities with regard to convex functions can be proved simply and directly. For instance, from the Jensen inequality as well as the Cauchy inequality and the Minkowski inequality, some important inequalities can be further deduced as follows.

Example 9 Let $\xi$ and $\eta$ be two fuzzy rough variables with $E\left[\xi^{2}\right]<\infty$ and $E\left[\eta^{2}\right]<\infty$. If the fuzzy variables $\xi(\lambda)$ and $\eta(\lambda)$ are independent for each $\lambda \in \Lambda$, then it follows from Example 8 and Theorem 11 that

$$
\sqrt{E\left[\xi^{2}\right] \cdot E\left[\eta^{2}\right]} \geq E[|\xi \eta|] \geq|E[\xi \eta]|
$$

and it follows from Theorem 10 and Theorem 11 that

$$
\sqrt{E\left[\xi^{2}\right]}+\sqrt{E\left[\eta^{2}\right]} \geq \sqrt{E\left[(\xi+\eta)^{2}\right]} \geq|E[\xi+\eta]| .
$$




\section{Critical values of fuzzy rough variable}

In this section, we first recall the concepts of the $(\gamma, \delta)$-optimistic value and the $(\gamma, \delta)$ pessimistic value of a fuzzy rough variable defined by Liu [5]. Then the linearity, monotonicity, and continuity of these critical values are discussed. It is shown that these mathematical properties, which have been discussed for fuzzy variables and rough variables by Liu [3] as well as for fuzzy random variables by Yang and Liu [19], are also valid for fuzzy rough variables in a similar way.

Definition 14 (Liu [5]) Let $\xi$ be a fuzzy rough variable, and $\gamma, \delta \in(0,1]$. Then

$$
\xi_{\text {sup }}(\gamma, \delta)=\sup \{r \mid \operatorname{Ch}\{\xi \geq r\}(\gamma) \geq \delta\}
$$

is called the $(\gamma, \delta)$-optimistic value of $\xi$, and

$$
\xi_{\text {inf }}(\gamma, \delta)=\inf \{r \mid \operatorname{Ch}\{\xi \leq r\}(\gamma) \geq \delta\}
$$

is called the $(\gamma, \delta)$-pessimistic value of $\xi$.

Theorem 12 (Liu [3]) Let $\xi$ be a fuzzy rough variable. Assume that $\xi_{\text {sup }}(\gamma, \delta)$ is the $(\gamma, \delta)$ optimistic value and $\xi_{\mathrm{inf}}(\gamma, \delta)$ is the $(\gamma, \delta)$-pessimistic value of $\xi$. If $\delta>0.5$, then we have

$$
\operatorname{Ch}\left\{\xi \leq \xi_{\text {inf }}(\gamma, \delta)\right\}(\gamma) \geq \delta, \quad \operatorname{Ch}\left\{\xi \geq \xi_{\text {sup }}(\gamma, \delta)\right\}(\gamma) \geq \delta .
$$

Theorem 13 (Linearity) Let $\xi_{\text {sup }}(\gamma, \delta)$ and $\xi_{\text {inf }}(\gamma, \delta)$ be the $(\gamma, \delta)$-optimistic and $(\gamma, \delta)$ pessimistic values of a fuzzy rough variable $\xi$, respectively. Then we have

(a) if $c \geq 0$, then $(c \xi)_{\text {sup }}(\gamma, \delta)=c \xi_{\text {sup }}(\gamma, \delta)$ and $(c \xi)_{\text {inf }}(\gamma, \delta)=c \xi_{\text {inf }}(\gamma, \delta)$;

(b) if $c<0$, then $(c \xi)_{\text {sup }}(\gamma, \delta)=c \xi_{\text {inf }}(\gamma, \delta)$ and $(c \xi)_{\text {inf }}(\gamma, \delta)=c \xi_{\text {sup }}(\gamma, \delta)$.

Proof (a) If $c=0$, then the part (a) is obvious. In the case of $c>0$, we have

$$
\begin{aligned}
(c \xi)_{\text {sup }}(\gamma, \delta) & =\sup \{r \mid \operatorname{Ch}\{c \xi \geq r\}(\gamma) \geq \delta\} \\
& =c \sup \{r / c \mid \operatorname{Ch}\{\xi \geq r / c\}(\gamma) \geq \delta\} \\
& =c \xi_{\text {sup }}(\gamma, \delta) .
\end{aligned}
$$

In a similar way we may prove $(c \xi)_{\text {inf }}(\gamma, \delta)=c \xi_{\text {inf }}(\gamma, \delta)$. In order to prove the part (b), it suffices to prove that $(-\xi)_{\text {sup }}(\gamma, \delta)=-\xi_{\text {inf }}(\gamma, \delta)$ and $(-\xi)_{\text {inf }}(\gamma, \delta)=-\xi_{\text {sup }}(\gamma, \delta)$. In fact, we have

$$
\begin{aligned}
(-\xi)_{\text {sup }}(\gamma, \delta) & =\sup \{r \mid \operatorname{Ch}\{-\xi \geq r\}(\gamma) \geq \delta\} \\
& =-\inf \{-r \mid \operatorname{Ch}\{\xi \leq-r\}(\gamma) \geq \delta\} \\
& =-\xi_{\text {inf }}(\gamma, \delta) .
\end{aligned}
$$

Similarly, we may prove that $(-\xi)_{\text {inf }}(\gamma, \delta)=-\xi_{\text {sup }}(\gamma, \delta)$. 
Theorem 14 (Monotonicity and continuity) Let $\xi_{\text {sup }}(\gamma, \delta)$ and $\xi_{\text {inf }}(\gamma, \delta)$ be the $(\gamma, \delta)$ optimistic and $(\gamma, \delta)$-pessimistic values of a fuzzy rough variable $\xi$, respectively. Then we have

(a) $\xi_{\text {sup }}(\gamma, \delta)$ is a decreasing and left-continuous function of $\gamma$ for each $\delta$;

(b) $\xi_{\text {sup }}(\gamma, \delta)$ is a decreasing and left-continuous function of $\delta$ for each $\gamma$;

(c) $\xi_{\text {inf }}(\gamma, \delta)$ is an increasing and left-continuous function of $\gamma$ for each $\delta$;

(d) $\xi_{\text {inf }}(\gamma, \delta)$ is an increasing and left-continuous function of $\delta$ for each $\gamma$.

Proof (a) For each fixed $\delta$, it is easy to prove that $\xi_{\text {sup }}(\gamma, \delta)$ is a decreasing function of $\gamma$. Next, we prove the left-continuity of $\xi_{\text {sup }}(\gamma, \delta)$ with respect to $\gamma$. Let $\gamma_{i}$ be an arbitrary sequence of numbers in $(0,1]$ such that $\gamma_{i} \uparrow \gamma$. Then $\xi_{\text {sup }}\left(\gamma_{i}, \delta\right)$ is a decreasing sequence. If the limitation is equal to $\xi_{\text {sup }}(\gamma, \delta)$, then the left-continuity is proved. Otherwise, we have

$$
\lim _{i \rightarrow \infty} \xi_{\text {sup }}\left(\gamma_{i}, \delta\right)>\xi_{\text {sup }}(\gamma, \delta)
$$

Letting $z^{*}=\left(\lim _{i \rightarrow \infty} \xi_{\text {sup }}\left(\gamma_{i}, \delta\right)+\xi_{\text {sup }}(\gamma, \delta)\right) / 2$, we have

$$
\xi_{\text {sup }}\left(\gamma_{i}, \delta\right)>z^{*}>\xi_{\text {sup }}(\gamma, \delta), \quad \forall i
$$

Then, for any given $\varepsilon>0$, there exists $A_{i}$ with $\operatorname{Tr}\left\{A_{i}\right\} \geq \gamma_{i}$ such that

$$
\inf _{\lambda \in A_{i}} \operatorname{Cr}\left\{\xi(\lambda) \geq z^{*}\right\} \geq \delta-\varepsilon, \quad \forall i
$$

Define $A^{*}=\bigcup_{i=1}^{\infty} A_{i}$. It is clear that $\operatorname{Tr}\left\{A^{*}\right\} \geq \operatorname{Tr}\left\{A_{i}\right\} \geq \gamma_{i}$. Letting $i \rightarrow \infty$, we get $\operatorname{Tr}\left\{A^{*}\right\} \geq \gamma$. Thus

$$
\operatorname{Ch}\left\{\xi \geq z^{*}\right\}(\gamma)=\sup _{\operatorname{Tr}\left\{A^{*}\right\} \geq \gamma} \inf _{\lambda \in A^{*}} \operatorname{Cr}\left\{\xi(\lambda) \geq z^{*}\right\} \geq \delta-\varepsilon
$$

Letting $\varepsilon \rightarrow 0$, we obtain $\operatorname{Ch}\left\{\xi \geq z^{*}\right\}(\gamma) \geq \delta$. Hence $z^{*} \leq \xi_{\text {sup }}(\gamma, \delta)$. A contradiction proves the left-continuity of $\xi_{\text {sup }}(\gamma, \delta)$ with respect to $\gamma$.

(b) For each fixed $\gamma$, it is easy to prove that $\xi_{\text {sup }}(\gamma, \delta)$ is a decreasing function of $\delta$. Next, we prove the left-continuity of $\xi_{\text {sup }}(\gamma, \delta)$ with respect to $\delta$. Let $\delta_{i}$ be an arbitrary sequence of numbers in $(0,1]$ such that $\delta_{i} \uparrow \delta$. Then $\xi_{\text {sup }}\left(\gamma, \delta_{i}\right)$ is a decreasing sequence. If the limitation is equal to $\xi_{\text {sup }}(\gamma, \delta)$, then the left-continuity is proved. Otherwise, we have

$$
\lim _{i \rightarrow \infty} \xi_{\text {sup }}\left(\gamma, \delta_{i}\right)>\xi_{\text {sup }}(\gamma, \delta)
$$

Letting $z^{*}=\left(\lim _{i \rightarrow \infty} \xi_{\text {sup }}\left(\gamma_{i}, \delta\right)+\xi_{\text {sup }}(\gamma, \delta)\right) / 2$, we have

$$
\xi_{\text {sup }}\left(\gamma, \delta_{i}\right)>z^{*}>\xi_{\text {sup }}(\gamma, \delta), \quad \forall i
$$

It follows from the definition of $\xi_{\text {sup }}\left(\gamma, \delta_{i}\right)$ that

$$
\operatorname{Ch}\left\{\xi \geq z^{*}\right\}(\gamma) \geq \delta_{i}, \quad \forall i
$$


Then, letting $i \rightarrow \infty$, we obtain

$$
\operatorname{Ch}\left\{\xi \geq z^{*}\right\}(\gamma) \geq \delta
$$

Hence $z^{*} \leq \xi_{\text {sup }}(\gamma, \delta)$. A contradiction proves the left-continuity of $\xi_{\text {sup }}(\gamma, \delta)$ with respect to $\delta$. The parts (c) and (d) may be proved similarly.

\section{Conclusions}

Based on previous study on inequalities and critical values in fuzzy set theory and rough set theory, this paper made a further study on fuzzy rough theory, and enriched the research area of this theory in the following two parts: (i) some inequalities in fuzzy rough theory were proved including the Markov inequality, the Chebyshev inequality, the Hölder inequality, the Minkowski inequality and the Jensen inequality, which are analogous to those of the fuzzy case and rough case; (ii) we explored the linearity, monotonicity and continuity of critical values of the fuzzy rough variable.

This paper discussed these inequalities as well as properties of the critical values in a theoretical way. However, the theorems and conclusions presented in this paper would also make an important contribution to practical applications of the fuzzy rough theory. Taking a decision system with fuzzy rough coefficients (e.g. [26, 27]) for instance, in order to get optimal solutions to a problem (modeled by a mathematical model with fuzzy rough coefficients), the objective functions as well as some constraints involving fuzzy rough variables should be analyzed. It is clear that the analyses of these functions and the development of a solving algorithm for the fuzzy rough model may benefit greatly from the inequalities and properties of the critical values presented in this paper.

Competing interests

The authors declare that they have no competing interests.

\section{Authors' contributions}

All authors contributed equally to the writing of this paper. All authors read and approved the final manuscript.

\section{Acknowledgements}

This work was supported in part by grants from the Ministry of Education Funded Project for Humanities and Social Sciences Research (No. 14YJC630124), the Shanghai Philosophy and Social Science Planning Project (No. 2014EGL002), and the Innovation Program of Shanghai Municipal Education Commission (No. 14YSO05).

Received: 10 April 2015 Accepted: 13 August 2015 Published online: 28 August 2015

References

1. Zadeh, LA: Fuzzy sets. Inf. Control 8, 338-353 (1965)

2. Kaufmann, A: Introduction to the Theory of Fuzzy Subsets. Academic Press, New York (1975)

3. Liu, B: Uncertainty Theory: An Introduction to Its Axiomatic Foundations. Springer, Berlin (2004)

4. Pawlak, Z: Rough sets. Int. J. Inf. Comput. Sci. 11, 341-356 (1982)

5. Liu, B: Theory and Practice of Uncertain Programming. Physica-Verlag, Heidelberg (2002)

6. Dubois, D, Prade, H: Rough fuzzy sets and fuzzy rough sets. Int. J. Gen. Syst. 17, 191-200 (1990)

7. Radzikowska, AM, Kerre, EE: A comparative study of fuzzy rough sets. Fuzzy Sets Syst. 126, 137-155 (2002)

8. Wu, WZ, Mi, JS, Zhang, WX: Generalized fuzzy rough sets. Inf. Sci. 151, 263-282 (2003)

9. DeCock, M, Cornelis, C, Kerre, EE: Fuzzy rough sets: the forgotten step. IEEE Trans. Fuzzy Syst. 15, 121-130 (2007)

10. Morsi, NN, Yakout, MM: Axiomatics for fuzzy rough sets. Fuzzy Sets Syst. 100, 327-342 (1998)

11. Jensen, R, Shen, Q: Fuzzy-rough sets assisted attribute selection. IEEE Trans. Fuzzy Syst. 15, 73-89 (2007)

12. Jensen, R, Shen, Q: New approaches to fuzzy-rough feature selection. IEEE Trans. Fuzzy Syst. 17, 824-838 (2009)

13. Chen, DG, Zhang, L, Zhao, SY, Hu, QG, Zhu, PF: A novel algorithm for finding reducts with fuzzy rough sets. IEEE Trans. Fuzzy Syst. 20, 385-389 (2012)

14. Jensen, R, Shen, Q: Fuzzy-rough attribute reduction with application to web categorization. Fuzzy Sets Syst. 141, 469-485 (2004)

15. Jensen, R, Shen, Q: Fuzzy-rough data reduction with ant colony optimization. Fuzzy Sets Syst. 149, 5-20 (2005)

16. Shen, Q, Richard, J: Selecting informative features with fuzzy-rough sets and its application for complex systems monitoring. Pattern Recognit. 37, 1351-1363 (2004) 
17. Lingras, P: Fuzzy-rough and rough-fuzzy serial combinations in neurocomputing. Neurocomputing 36, $29-44$ (2001)

18. Liu, B: Inequalities and convergence concepts of fuzzy and rough variables. Fuzzy Optim. Decis. Mak. 2, 87-100 (2003)

19. Yang, LX, Liu, B: On inequalities and critical values of fuzzy random variables. Int. J. Uncertain. Fuzziness Knowl.-Based Syst. 13, 163-175 (2005)

20. Zadeh, LA: Fuzzy sets as a basis for a theory of possibility. Fuzzy Sets Syst. 1, 3-28 (1978)

21. Zadeh, LA: A theory of approximate reasoning. In: Hayes, J, Michie, D, Thrall, RM (eds.) Mathematical Frontiers of the Social and Policy Sciences, pp. 69-129. Westview Press, Boulder (1979)

22. Dubois, D, Prade, H: Possibility Theory: An Approach to Computerized Processing of Uncertainty. Plenum, New York (1988)

23. Dubois, D, Prade, H: Fuzzy numbers: an overview. In: Bezdek, JC (ed.) Analysis of Fuzzy Information, vol. 2, pp. 3-39. CRC Press, Boca Raton (1988)

24. Liu, B, Liu, YK: Expected value of fuzzy variable and fuzzy expected value models. IEEE Trans. Fuzzy Syst. 10, 445-450 (2002)

25. Liu, Y, Gao, J: The independence of fuzzy variables in credibility theory and its applications. Int. J. Uncertain. Fuzziness Knowl.-Based Syst. 15, 1-20 (2007)

26. Mondal, M, Maity, AK, Maiti, MK, Maiti, M: A production-repairing inventory model with fuzzy rough coefficients under inflation and time value of money. Appl. Math. Model. 37, 3200-3215 (2013)

27. $\mathrm{Xu}$, J, Zhao, L: A multi-objective decision-making model with fuzzy rough coefficients and its application to the inventory problem. Inf. Sci. 180, 679-696 (2010)

\section{Submit your manuscript to a SpringerOpen ${ }^{\circ}$ journal and benefit from:}

- Convenient online submission

Rigorous peer review

Immediate publication on acceptance

Open access: articles freely available online

- High visibility within the field

- Retaining the copyright to your article 